\title{
Patient Demographics at an Urban Acupuncture Teaching Clinic
}

Abstract

The primary aim of our study was to describe the demographic characteristics of acupuncture patients in an urban teaching clinic and the diagnoses of those patients. The demographics of those seeking acupuncture treatments at the University of Bridgeport Acupuncture Institute clinic are primarily female, mostly Caucasian, with an average age of 55.7 years. Traditional Chinese Medicine (TCM) patient demographics at an urban teaching clinic match those in other settings around the world

Keywords: Urban acupuncture; Traditional chinese medicine; TCM clinic data; Electronic health records; EHR system

Abbreviations: TCM: Traditional Chinese Medicine; EHRs: Electronic Health Records; CGM: Compagnie Générale Maritime

\section{Background}

The demographics of those seeking acupuncture and related Traditional Chinese Medicine (TCM) treatments in a variety of teaching centers has been discussed in several articles [1-9]. The demographics of acupuncture patients in these studies were similar. In the US, the majority of patients were Caucasian females [1-4]. In Korean settings, the majority of patients were Asian with less of a gap between the genders $[5,6]$. Recognizing that TCM clinical experiences change over time and practice varies in different settings, there is a need to conduct evaluations of TCM clinic data to better inform TCM education and clinical training in the US.

The primary aim of our study was to describe the demographic characteristics of acupuncture patients in an urban teaching clinic and the diagnoses (identified by ICD10 coding) of those patients. Our secondary aim was to compare these profiles of acupuncture patients with those done in other studies and other types of treatment settings.

\section{Methods}

This study was a retrospective review of the electronic health records (EHRs) of patients who had received at least one session of acupuncture at the UB Acupuncture Clinics during one academic year (2 semesters). We performed an electronic chart review at the University of Bridgeport Acupuncture Institute teaching clinic, part of the "UB Clinics" in Bridgeport, CT. Information that existed in automatically extractable form was collected in a predefined Excel spreadsheet. Unidentified patient data of those who had received acupuncture (as identified as being billed with the CPT code 97810) were extracted from the Electronic health Record (EHR). Specifically, patient ID numbers, gender, age, selfidentified race information and ICD10 diagnoses were extracted to an Excel spreadsheet. This information existed in automatically extractable form due to the use of an electronic health record

Mini Review
Volume 8 Issue 6 - 2017
Jennifer Brett* and Peter Tournas
Naturopathic Physician \& Acupuncturist, University of
Bridgeport Acupuncture Institute, USA
*Corresponding author: Jennifer Brett, Naturopathic
Physician \& Acupuncturist, University of Bridgeport
Acupuncture Institute, 60 Lafayette Street, Bridgeport, CT
06601, USA, Tel: 203-576-4122; Email:
jbrett@bridgeport.edu
Received: September 13, 2017 | Published: September 18,
2017

(EHR). After extraction, the data was analyzed for demographic and diagnostic groupings.

The primary diagnoses (ICD 10 Codes) were based on the patient's history, clinical examination and previously diagnosed diseases. All ICD 10 codes were selected by the student interns and verified by the supervising clinicians. The Senior Clinical Administrator for the UB Clinics, who was not involved in this study, oversaw the data extraction. All extracted data from the EHRs database was done by the company who provides the EHR system (CGM).

\section{Findings}

During the 2016 Academic year (9/12/15 to 6/15/16) there were 3836 Acupuncture patient visits in the UB Clinics. Of these, a majority were female, with an average age in their fifties and a chief complaint of pain. This compares favorably with the previous demographic surveys.

\begin{tabular}{|c|c|c|c|c|}
\hline \multicolumn{2}{|c|}{ Study Location } & \multicolumn{2}{|c|}{$\%$ Female } & $\%$ Male \\
\hline \multicolumn{2}{|c|}{ UB } & \multicolumn{2}{|l|}{73} & 27 \\
\hline \multicolumn{2}{|c|}{ Other US Studies (1-4) } & \multicolumn{2}{|l|}{70} & 30 \\
\hline \multicolumn{2}{|c|}{ UK (8) } & \multicolumn{2}{|l|}{74} & 26 \\
\hline \multicolumn{2}{|c|}{ Korea $(5,6)$} & \multicolumn{2}{|l|}{57} & 43 \\
\hline & UB Clinics & USA & UK & Korea \\
\hline Under 21 & 1.5 & & & \\
\hline $21-40$ & 16.9 & 19.2 & 19.5 & 25.4 \\
\hline $41-50$ & 16.5 & 21.7 & 22.4 & 25.9 \\
\hline $51-60$ & 24.6 & 26.5 & 28.7 & 27.6 \\
\hline $61-80$ & 36.3 & 28.9 & 26.2 & 19.4 \\
\hline over 80 years & 4.2 & 3.7 & 3.2 & 1.7 \\
\hline Average & 55.7 & 42.5 & 51 & 52.0 \\
\hline
\end{tabular}




\begin{tabular}{|c|c|}
\hline $\begin{array}{c}\text { UB Clinics Acupuncture Patients } \\
\text { Self-Identified Race }\end{array}$ & $\%$ \\
\hline Asian & 5.6 \\
\hline African American & 7.4 \\
\hline Caucasian/non-Latino & 65.9 \\
\hline Other & 19.9 \\
\hline
\end{tabular}

Diseases diagnosed by clinicians at the UB Clinics TCM teaching clinic were grouped by ICD10 category. During patient assessment, those treated in the UB Clinics with acupuncture had the following top primary groups of diagnoses:

\begin{tabular}{|c|c|}
\hline Category of Diagnosis & Percentage (\%) \\
\hline Musculoskeletal Diagnoses & 49.9 \\
\hline General Wellness & 12.9 \\
\hline Nervous System Disorders & 8.7 \\
\hline Mental/Behavioral Diseases & 6.4 \\
\hline Digestive Disorders/Illnesses & 6.3 \\
\hline Female Genitourinary Diseases & 4.1 \\
\hline Other, Misc. & 11.7 \\
\hline Total & 100 \\
\hline
\end{tabular}

The number of visits each individual patient was seen during this timeframe ranged from single visits to over 11 visits per year. While both men and women clustered in the 2 to 5 visit range, suggestive of people coming to be treated for a specific complaint, women were more likely to be "super users" [9] of acupuncture at a rate of $16.4 \%$ compared to $7 \%$ of men being seen more than 10 times per year (Figure 1).

\begin{tabular}{|c|c|c|}
\hline \# of Visits to UB Clinics & Female & Male \\
\hline 1 & $28.40 \%$ & $29.60 \%$ \\
\hline 2 to 5 & $44.80 \%$ & $49.30 \%$ \\
\hline 6 to 10 & $10.30 \%$ & $14.10 \%$ \\
\hline 11 plus & $16.40 \%$ & $7 \%$ \\
\hline
\end{tabular}

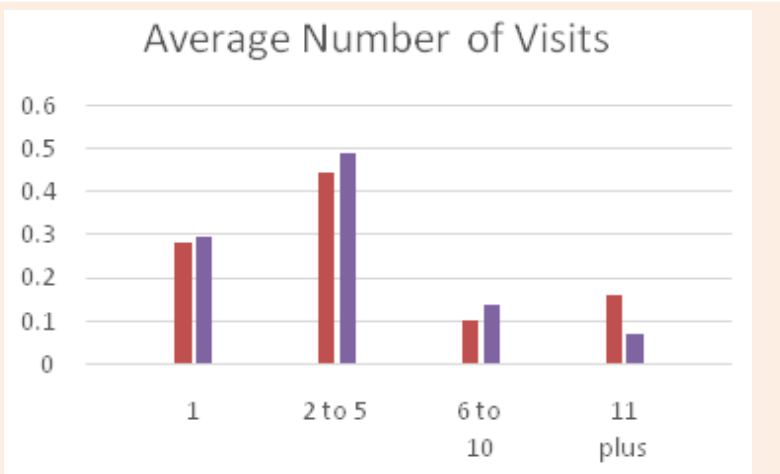

- FEMALE M MALE

Figure 1: The number of visits each individual patient.

\section{Discussion}

The UB Clinics is a teaching clinic associated with a university situated near the Long Island Sound in the south end of Bridgeport Connecticut, and low-income urban area with a median per capita income of $\$ \$ 22,422$. In Connecticut, many healthcare insurers cover acupuncture treatments for pain diagnoses. However, acupuncture is not covered by Medicare or Medicaid services in CT. The comparatively low cost of our acupuncture services is probably one factor in seeing patients in our teaching clinic, especially for those over 65 years of age. It is also possible that people seeking more information about acupuncture "try" the UB Clinics because it is a teaching clinic.

The patients' demographic characteristics for the UB Clinics were similar to other published demographic studies of acupuncture. Namely, our patients were primarily female, mostly Caucasian, with an average age of 55.7 years. The average number of individual patient visits was four [4] during this time period. Diseases diagnosed by our clinicians at the TCM teaching clinic were primarily those of the musculoskeletal system.

This study compares favorably with previous acupuncture studies in various treatment settings.

Instructors teaching acupuncture in the US need to be sure student interns are prepared to treat patients with musculoskeletal pain and neurological disorders with special emphasis on those over 50 years of age.

\section{Conclusion}

The demographics of those seeking acupuncture treatments at the University of Bridgeport Acupuncture Institute clinic are primarily female, mostly Caucasian, with an average age of 55.7 years. Traditional Chinese Medicine (TCM) patient demographics at an urban teaching clinic match those in other settings around the world.

\section{References}

1. Soliday E, Hapke P (2014) Characteristics of a U.S. obstetric acupuncture clinic patient sample. Explore (NY) 10(2): 109-114.

2. Marx BL, Rubin LH, Milley R, Hammerschlag R, Ackerman DL (2016) A prospective patient-centered data collection program at an acupuncture and oriental medicine teaching clinic. J Altern Complement Med 19(5): 410-415.

3. Hsu KY, Dunn JE, Bradshaw YS, Conboy L (2017) Lessons from an acupuncture teaching clinic: patient characteristics and pain management effectiveness. Explore (NY) 10(5): 284-293.

4. Maiers M, McKenzie E, Evans R, McKenzie M (2008) Patient outcomes at a traditional Chinese medicine teaching clinic: a prospective data collection project. J Altern Complement Med 14(9): 1083-1088.

5. Yun KJ, Lee JA, Choi J, Ko MM, Lee CK, et al. (2015) A Retrospective Analysis of Patients' Conditions Using Acupuncture in a Traditional Korean Medicine Hospital. Evid Based Complement Alternat Med 2015: 103683.

6. Kim KH, Kim YR, Noh SH, Kang KW, Kim JK, et al. (2013) Use 
of acupuncture for pain management in an academic Korean medicine hospital: a retrospective review of electronic medical records. Acupunct Med 31(2): 228-134.

7. Jiang J, Pang W, Gu T, King C, Yin JK (2016) Critical Review of Data Evaluation in Teaching Clinics of Traditional Chinese Medicine Outside China: Implications for Education. Explore (NY) 12(3): 188-195.
8. MacPherson H, Sinclair-Lian N, Thomas K (2016) Patients seeking care from acupuncture practitioners in the UK: A national survey. Complement Ther Med 14(1): 20-30.

9. Cooper F, Marx BL, Lee TL, Espesete D (2017) Super-Users at an Acupuncture and Oriental Medicine Teaching Clinic: Demographics and Unique Clinical Characteristics. J Altern Complement Med 23(3): 222-226. 